\title{
LEVELS OF ANALYSIS IN MOTOR CONTROL
}

RICHARD W. PEW

The University of Michigan, Ann Arbor, Mich. 48104 (U.S.A.)

\section{SUMMARY}

The analysis of skilled performance must proceed at many levels. As illustrated in the two experiments presented here, what we observe in human skilled behavior is the rich intermingling of these various levels of control as a function of the task demands, the state of learning of the subject and the constraints imposed on the task and the subject by the environment. While we should be interested in the neurophysiological correlates of such behavior we still have a long way to go even to understand what to expect in terms of the behavior itself. The job of the researcher is different depending on the level of analysis at which he is interested, but a general theory of skill acquisition will only result from consideration of the ramifications of a multilevel process-oriented description of skilled performance.

\section{RÉSUMÉ}

L'étude de la performance motrice doit procéder de bien des niveaux d'analyse. Comme l'illustrent les deux expériences présentées ici, ce que nous observons dans le comportement humain, c'est l'intrication de ces divers niveaux de contrôle en fonction de la tâche proposée, de ce que sait le sujet et des contraintes imposées par l'environnement à l'une et à l'autre. Bien que nous soyons intéressés par les correlats neurophysiologiques de tels comportements nous avons encore un long chemin à parcourir pour comprendre ce que nous pouvons déjà espérer atteindre en terme de comportement. La tâche du chercheur est différente suivant le niveau d'analyse auquel il s'intéresse, mais une théorie générale de l'acquisition des habilités motrices ne peut 
résulter que de la prise en considération des ramifications d'une analyse des processus mis en jeu aux divers niveaux de description de la performance motrice.

The performance of skilled movement has been of considerable interest to psychologists in recent years, yet we still lack sufficient information to be able to describe adequately the mechanisms that characterize performance of learned patterns of movement. In this paper I want to introduce two experiments that address this question, and then to present a multi-level representation that may provide a starting point for the elaboration of a theory of skilled performance.

\section{Performance effects of extended practice}

The first experiment addresses the question of differential learning of predictable and unpredictable sequences of movement. It was conducted by $C$. D. Wickens and the author. The subject rested his arm on a control stick pivoted at the elbow that moved left and right in the horizontal plane. He used it to perform a pursuit tracking task. Movement of the stick controlled the movement of a cursor on a $30.48 \mathrm{~cm}$ (12 in.) oscilloscope (CRT). Angular rotation of $2.36^{\circ}$ of the stick produced $1 \mathrm{~cm}$ deflection of the cursor on the CRT that was placed approximately $70 \mathrm{~cm}$ from the subject's eyes. The control stick had negligible inertia and damping and had a spring constant of $1.05 \mathrm{~g} /$ degree.

From the subject's point of view the target pattern to be followed was a smooth, random appearing signal that was produced by passing a waveform consisting of constant-amplitude positive and negative steps of varying durations through a 1.0 $\mathrm{rad} / \mathrm{sec}(0.016 \mathrm{~Hz})$ cutoff third-order Butterworth filter. The signal had a Gaussian amplitude distribution, and each trial lasted $65 \mathrm{sec}$. From the point of view of the experimenter the signal was divided into three 20 -sec segments plus an additional lead-in period of $5 \mathrm{sec}$ before scoring began. On each trial the first and last $20 \mathrm{sec}$ were different samples of the same random process. However, the middle $20 \mathrm{sec}$ was the same sample of the random process on every trial except during test periods to be described in a moment. Fig. 1 presents the signal to be followed on 5 different trials superimposed on top of each other. It is easy to see that the first and last segments are different each time, but the middle segment is always the same. Five male subjects who volunteered to serve in paid experiments simply tried to track this signal for $1 \mathrm{~h}$ (24 trials), each day for 16 days. At various points during this period of practice, experimental manipulations were introduced as described in the subsequent paragraphs.

First, it was established, as shown in Fig. 2, that, initially, average performance of the 5 subjects as measured by the integrated-absolute-error score on the 3 segments separately, was essentially the same, but as a function of practice the B or repeated segment performance gradually improved faster than on the random $\mathrm{A}$ and $\mathrm{C}$ segments. By day 6 the difference between the random and repeated segments was reliably different and continued to get bigger thereafter. The subjects were able to take advantage of the predictability of the repeated segment to improve their performance over that of its random counterparts. There were also consistent and reliable differences 


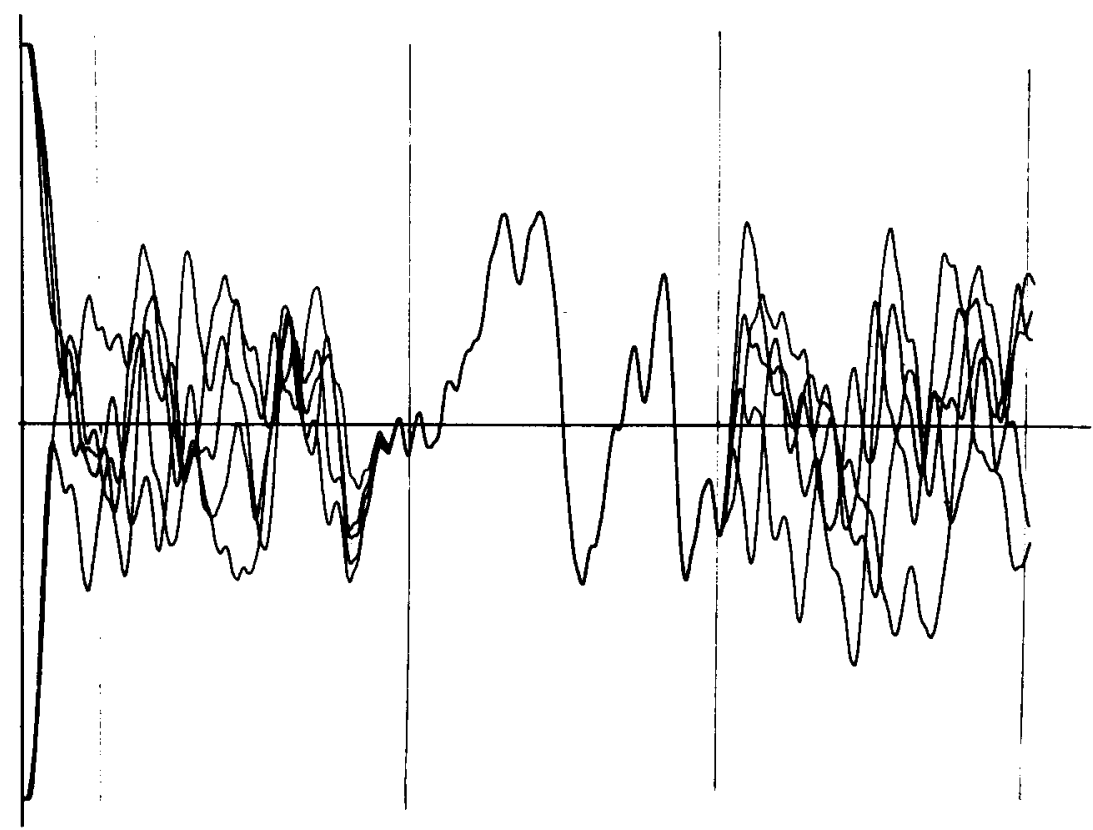

Fig. 1. Illustration of the superposition of 5 samples of the stimulus patterns to be followed. The amplitude of the movement is shown on the ordinate and time is shown on the abscissa increasing from left to right. The time between vertical lines is $20 \mathrm{sec}$. The initial $5 \mathrm{sec}$ at the left of the figure was not scored.

between performance of the A and C segments, both of which were random. At least a part of this difference is attributable to an experimental artifact. In order to ensure that the repeated signal was identical throughout the 20 -sec period, the repeated step sequence was introduced $5 \mathrm{sec}$ prior to the beginning of the repeated segment scoring period in order for the transients of the analog filter to die out. However, as can be seen from Fig. 1, the signal is, on the average, identical for some brief period prior to the B segment measurement period. Thus a portion of the A segment, perhaps $5 \%$, is also identical on every trial, giving the A segment a performance advantage over the $C$ segment that is reflected in the data. We estimate that this can account for a performance difference of about $5 \%$ and the remaining 1 or $2 \%$ we can only attribute to genuine differences between the beginning and end of the trial.

At the conclusion of practice on day 11 we conducted an interview with the subject that was designed to elicit from him whether he could report on the presence or character of the repeated segment. The interview began with a very general question concerning the subject's experience in the experiment, became systematically more specific until the last question which asked, 'The middle $20 \mathrm{sec}$ of every trial was exactly repeated on every trial. Did you notice that?'. Three of the 5 subjects answered no to the last question and the two that had reported on its repeated nature earlier had only a diffuse idea of the nature of the repetition. One stated that he thought it repeated on about two-thirds of the trials, and another said the repeats did not seem localized but could occur anywhere in the trial period. It seemed clear that whatever the subject 


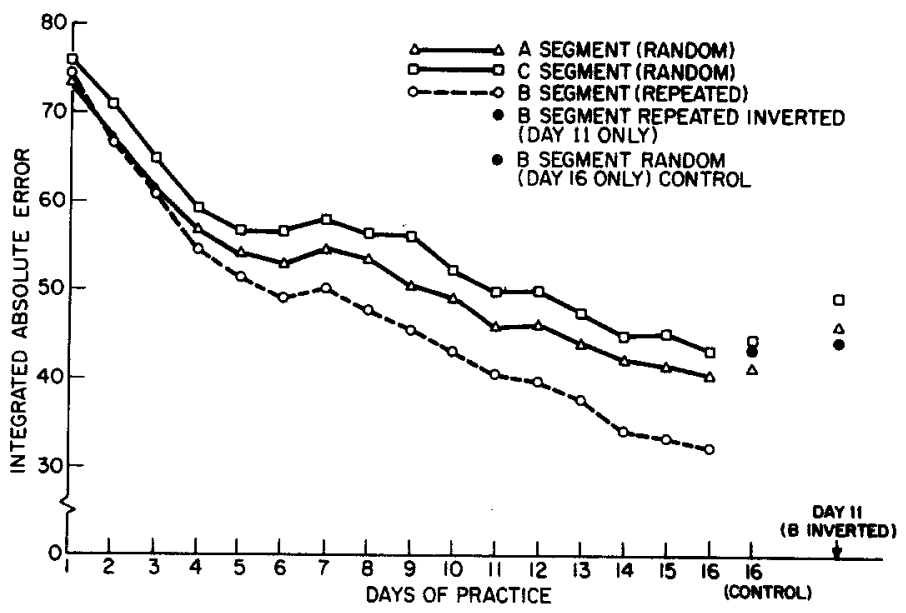

Fig. 2. Error performance as a function of practice shown separately for the three 20-sec segments of the pattern in experiment 1 . Also shown is the performance when the repeated segment was reversed on day 11 , together with a control condition (not mentioned in the text) in which the repeated segment was replaced by a new random segment on day 16 and the performance difference was eliminated.

was doing he was not modifying his behavior on the basis of reportable knowledge about the character of the repeated segment.

Two further manipulations were designed to assess the nature of the information stored about the repeated segment that gave it its performance advantage. At the end of day 11 but before the interview, the subjects were administered 8 trials during which everything about the trial was the same except that the repeated segment was exactly inverted. Whenever the subject had to move to the right before, now he had to move to the left and vice versa. If what was being learned was a motor program that has the character of a phonograph record or fixed tape, a 'carved-in' automatic series of movements, one might expect that reversing the signal might make performance on the B segment worse than the random A and C segments. In fact, that did not happen. The average performance on these trials for the 3 segments respectively is shown in Fig. 2 at the left of the figure.

The B segment was reliably better than the average of the $\mathrm{A}$ and $\mathrm{C}$ segments together $(t=2.53, P<0.02)$, and reliably worse $(t=3.05, P<0.01)$ than the unreversed B-segment performance on the same day. Whatever it is that was learned appeared to generalize at least to some extent to corresponding patterns symmetrically reversed. That is, symmetry need not be preserved to preserve the significant features of the pattern that have been learned.

Finally, on days 6,11,12 and 16,20 trials were administered instead of 24 and on half of them the subjects performed a memory task concurrently with the tracking task to assess the differential effects of the extra memory load on tracking of the repeated and nonrepeated segments. The memory task required reporting back a sequence of auditorily presented words at 1 per $2 \mathrm{sec}$ with a lag of one word. That is, each time they heard a new word they reported out the word they had heard just before it. 
A common definition of overlearning or 'automation' of a movement sequence asserts that an automated movement requires less 'attention' or 'processing capacity' to complete its execution accurately. It is interesting to note therefore in this experiment that while a significant tracking decrement occurred for all 3 segments, there was no tendency for the repeated segment to be more resistant to the effects of the extra memory load. In fact, the trend was the other way. There was a somewhat greater decrement in tracking performance for the repeated segment than for its random counterparts and the memory task performance, while overall quite good, was significantly worse for the repeated than the non-repeated segments $\left(\chi_{2}^{2}=0.036\right.$, $P>0.05$ ). Whatever the subjects learned in this experiment appears to require sustained attention for its effects to be manifest. These experimental manipulations illustrate particularly well the power of this paradigm as a tool for the further elaboration of the nature of performance changes brought about by extended specific practice.

\section{Learning memorized movement patterns}

The second experiment is a portion of T. R. Armstrong's thesis research ${ }^{2}$ and was designed to examine alternative ways to teach a subject to execute a particular irregular movement pattern from memory. The pattern was defined more or less arbitrarily to be approximately $4 \mathrm{sec}$ long and to involve 4 reversals of arm direction. The subjects produced the pattern by moving the arm control stick described previously. The maximum amplitude of the movement as measured at the hand grip of the stick was an arc of $61 \mathrm{~cm}$.

Three experimental training conditions were compared with a control group that attempted to produce the movement from memory on 24 blocks of 10 trials, distributed equally over 4 days (IAE group). After each trial all subjects were told their integrated absolute error score, the average deviation of the pattern they produced from the pattern they were trying to produce. After each block of 10 trials they were shown an $\mathrm{X}-\mathrm{Y}$ recording of what they had done in comparison to what they should have done and then began the next block of 10 trials.

Subjects in the first experimental condition (CRT group) practiced the task as a pursuit tracking task in which the pattern to be followed was presented as a moving target on a $30.48 \mathrm{~cm}$ (12 in.) CRT. A cursor was driven by movement of the control stick and the subject was instructed to keep the two symbols superimposed. The second group (GUI group) had their arm driven through the pattern by a computer-controlled torque motor mounted in the base of the stick. Their instructions during the practice trials were to try to anticipate the movement of the stick. The third experimental group (STK group) tried to produce the pattern from memory during the practice trials, however, they received some proprioceptive feedback about their errors. Whenever they deviated from the edges of a target zone $5.71 \mathrm{~cm}(2.25 \mathrm{in}$.) wide, centered on the target path to be followed, they felt a force gradient that tended to return their arm to the target zone. To the subjects it felt like a moderately heavy spring pulling them back on target. Within the target zone they felt no spring forces.

Each of the experimental groups worked in their respective practice conditions for three 10-trial blocks and then performed for one block under the same conditions 
as the control group. This sequence of 4 blocks was repeated 6 times during the 3-day period and then on the fourth day all subjects performed in the control condition for an additional six 10-trial blocks. Thus the experimental subjects alternated between practice and test conditions during the 4 daily sessions, while the control group always worked under the condition to be tested.

Fig. 3 shows the informative but somewhat discouraging results of this experiment from the point of view of its intended purpose. While the experimental groups performed better with the assistance they were provided during practice, at no time were they ever better than the control group on the test conditions, and by the fourth test day there were no significant differences among any of the 4 groups.

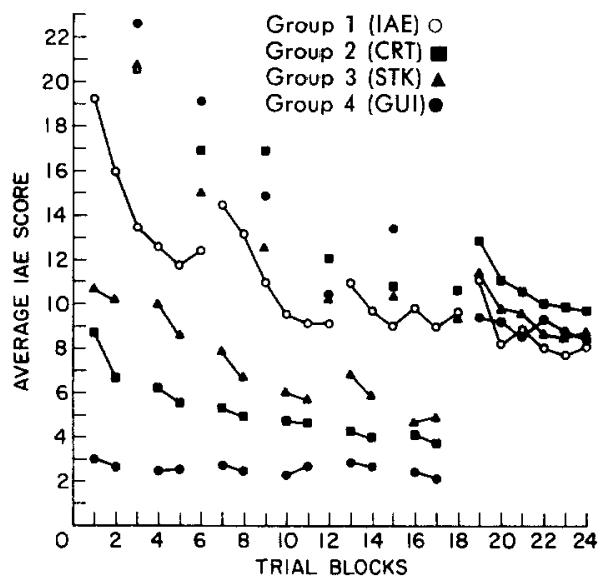

Fig. 3. Error performance as a function of practice and test conditions in experiment II. The symbols referring to each group are identified in the text.

All the experimental training conditions were designed to provide immediate information that would aid in accurate performance during the practice trials. This experiment, together with two earlier ones designed with a similar purpose ${ }^{2,8}$, leads one to the conclusion that such corrective information contributes little if anything to the learning of the movement pattern to be performed from memory. We conclude that whatever is learned about such patterns of movement depends on a more global picture of the movement pattern as a whole, than is provided by this kind of corrective feedback. Said another way; subjects need information which they can use to improve their performance on subsequent trials, not information that contributes to better performance on the trial in progress. The level of control at which attention is focussed appears to be much more important than the modality or nature of the corrective feedback itself.

\section{A multi-level perspective of skills}

It is difficult to explain the results of these experiments in terms of a single level of analysis of performance of the motor system. One should think instead of a hier- 
archy of levels of control of which three examples will be presented here. A more detailed presentation and discussion of these levels of analysis are contained in ref. 7 .

The first and most fundamental level is typical of that needed to represent the data presented in many of the papers in this symposium, especially that of the discontinuous movements described by Professor Brooks ${ }^{4}$.

Performance early in practice in the first experiment described above and late in practice for the random segments can be accommodated adequately by an elementary servomechanism such as that described by McRuer and Jex ${ }^{6}$, Lamey and Westcott ${ }^{5}$ or several others. In contrast with servomodels directed to the description of reflexive control, these models operate on visually perceived error and imply at least some central (i.e., cortical) involvement. They incorporate a simple error detector, a mechanism for converting the weighted sum of visually defined position and velocity information into motor commands and an approximation to the muscle system that translates motor command signals into actual movements.

Such a low level corrective servo system, however, is inadequate to explain the performance on the repeated segment in experiment I. In this case, although performance is still well defined by the target pattern to be followed, the subject is capable of superseding the elementary control loop to generate more complex patterned outputs lasting longer than simple corrections and to monitor the correspondence between the generated pattern and the desired pattern using more sophisticated error detection mechanisms.

The two levels described thus far may be thought of as analogous to the saccadic and pursuit eye movement systems. The saccadic system being the discrete error correction system and the pursuit system corresponding to a higher level tracking system that operates on the input signal directly and formulates commands on that basis to move the eye at constant velocities.

Tracking tasks like that employed in experiment I provide a well-defined input pattern to be followed. However, the full range of skilled movement implies something more. It implies that after practice the input can be formulated internally on the basis of considerations of the goal of the movement, the stimulating conditions at the time the movement is initiated and the dynamic state of the subject at that instant. While experiment II does not capture the full richness of 'voluntary' movement in the sense that the movement pattern required was predefined in a way typical unconstrained movements are not, this experiment does focus our attention on a very relevant question for the analysis of self-initiated movements namely: What is it that is stored when one has acquired the ability to call up movement patterns appropriate to riding a bicycle, throwing a ball at a moving target or performing industrial assembly tasks?

This then is the third and most complex level of analysis that I want to propose: the performance of goal oriented, self-initiated movements. Bernstein ${ }^{3}$ and Anokhin ${ }^{1}$ provide a conceptualization of this problem and I have addressed some of the issues 7 but we have a long way to go before we can synthesize a model of skilled behavior at this level that would be capable of mimicking human performance in any realistic sense. There are just too many degrees of freedom remaining. 
Experiment II was conducted with support from the U. S. National Aeronautics and Space Administration under Contract NSR-23-005-364. Experiment I and the preparation of this paper were supported by the Advanced Research Projects Agency of the U. S. Department of Defense and monitored by the Air Force Office of Scientific Research under Contract No. F44620-72-C-0019.

1 Anokhin, P. K., Cybernetics and the integrative activity of the brain. In M. COLE AND I. MaltzMAN (Eds.), A Handbook of Contemporary Soviet Psychology, Basic Books, New York, 1969, Ch. 30.

2 Armstrong, T. R., Training for the production of memorized movement patterns, Human Performance Center Technical Report No. 26, University of Michigan, August, 1970.

3 Bernstein, N., The Co-ordination and Regulation of Movements, Pergamon Press, Oxford, 1967.

4 Brooks, V. B., The programming of limb movements and its neural correlates: Introductory lecture to session III, Brain Research, 71 (1974) 299-308.

5 Lamey, L. P., AND Wescott, J. H., The stimulation of human operator tracking using an intermittent model, International Congress on Human Factors in Electronics, Long Beach, California, May, 1962.

6 MCRuer, D. T., AND JEx, H. R., A review of quasi-linear pilot models, IEEE Trans. Hum. Factors Electronics, HFE-8 (1967) 231-249.

7 PEw, R. W., Human perceptual-motor performance. In B. Kavtowitz (Ed.), Human Information Processing: Tutorials in Performance and Cognition, Lawrence Erlbaum Associates, Potomac, Md., 1974, Ch. 1.

8 RoBb, M., AND PEw, R. W., Skill training for the production of a memorized movement pattern, U.S. National Aeronautics and Space Administration Contractor Report 1251, 1968. 